\title{
Comparison of Extraction Socket Healing in Non-Diabetic, Prediabetic, and Type 2 Diabetic Patients
}

This article was published in the following Dove Press journal: Clinical, Cosmetic and Investigational Dentistry

\author{
Srikanth Gadicherla' \\ Komal Smriti ${ }^{2}$ \\ Sreea Roy' \\ Kalyana- \\ Chakravarthy Pentapati (D) $^{3}$ \\ Jyotsna Rajan' \\ Apoorva Walia' \\ 'Department of Oral and Maxillofacial \\ Surgery, Manipal College of Dental \\ Sciences, Manipal, Manipal Academy of \\ Higher Education, Manipal, Karnataka, \\ India; ${ }^{2}$ Department of Oral Medicine and \\ Radiology, Manipal College of Dental \\ Sciences, Manipal, Manipal Academy of \\ Higher Education, Manipal, Karnataka, \\ India; ${ }^{3}$ Department of Public Health \\ Dentistry, Manipal College of Dental \\ Sciences, Manipal, Manipal Academy of \\ Higher Education, Manipal, Karnataka, \\ India
}

Objective: To compare the healing of extraction socket among non-diabetic, prediabetic, and diabetic patients.

Materials and Methods: A single-center prospective observational study was conducted. Glycated hemoglobin and random blood glucose were recorded for all the participants before the procedure. A trained and calibrated examiner evaluated the socket size on postoperative days 0 and 7. Postoperative pain ( $\mathrm{PoP}$ ), discharge, swelling, infection, erythema, dry socket, and the number of analgesics were also recorded.

Results: A total of 100 participants completed this study with a mean age of $54.7 \pm 12.11$. There was no significant difference in the mean socket size among the three study groups on day $0(\mathrm{P}=0.101)$. However, there was a significant difference in the mean socket size on day 7 among the three groups. A post hoc test showed that the diabetic group had a larger socket size than the non-diabetic group $(\mathrm{P}=0.011)$. Complications like swelling and infection were more in the diabetic group. There was no significant difference in the mean number of analgesics among the three groups $(\mathrm{P}=0.169)$. The adjusted means for the socket size on postoperative day 7 was significantly higher for diabetic than the non-diabetic group.

Conclusion: The socket dimension was larger on postoperative day 7 in people with diabetes which suggested delayed healing without persistent complications. Dental extractions can be performed safely in optimally controlled diabetic patients with minimal complications.

Keywords: diabetes, delayed, extraction, healing, outcomes

\section{Introduction}

Diabetes mellitus (DM) is a metabolic disorder of multiple etiology, characterized by chronic hyperglycemia with disturbances of carbohydrate, fat and protein metabolism resulting from defects in insulin secretion, insulin action, or both. ${ }^{1}$ It is either characterized by deficient insulin secretion or peripheral insulin insensitivity or both, which can give rise to several metabolic disorders. ${ }^{2} \mathrm{DM}$ is rapidly gaining the status of a potential epidemic in India, with more than 62 million people affected. By 2030, India would have 79.4 million people with DM. ${ }^{3,4}$ Many complications (macrovascular and microvascular) have been attributed to be caused by diabetes. There is a pronounced alteration in the microvascular circulation, which results in a reduced inflammatory response. It can decrease leukocyte migration, tissue perfusion, and impaired hyperemia. Overall, the delivery of nutrients and removal of metabolic by-products are affected. Due to the microvascular changes, people with diabetes are at increased risk of postoperative
Correspondence: Kalyana-Chakravarthy Pentapati

Department of Public Health Dentistry

Manipal College of Dental Sciences,

Manipal, Manipal Academy of Higher

Education, Manipal, Karnataka, India

Tel +9l-99l-603-6303

Email drkalyan8I@gmail.com 
infection and delayed wound healing., 5ata from several reports suggest that people with diabetes are at high risk of periodontal disease and tooth loss. ${ }^{7-9}$ Previous research has not reported delayed wound healing and increased postoperative infections after tooth extraction. ${ }^{10-13}$ Although there exists a systematic review on the management of tooth extraction in diabetic patients, there is a paucity of literature on the outcomes of tooth extraction in people with diabetes. ${ }^{14}$

Due to lack of proper documented literature, minor oral surgical treatments including extractions are deferred by many oral health professionals in diabetic patients citing reasons like delayed wound healing and increased risk of postoperative infection. In such situations, physician consultation is warranted amounting to unnecessary delay in treatment and also unnecessary administration of preoperative antibiotics and analgesics. All of these factors can have significant implications and negative impacts on the quality of life of the individuals.

The present study aimed to evaluate the effect of diabetes on healing of extraction socket in a population sample in India. Various parameters like extraction socket size, postoperative pain (PoP), discharge, swelling, infection, erythema, the occurrence of dry socket, and the number of analgesics consumed over a period of one week were assessed.

\section{Materials and Methods}

\section{Study Design and Sample}

A prospective observational study was carried out in the Department of Oral and Maxillofacial surgery, Manipal College of Dental Sciences, Manipal, Karnataka, India. The study protocol was approved by the Institutional Ethical committee, Kasturba Hospital, Manipal. A total of 275 participants (non-diabetic, prediabetic, and diabetics) were screened, out of which 120 patients qualified the inclusion criteria. They were invited to participate in the study, out of which 104 patients consented (Figure 1). In view of the possible unequal enrolment of the participants and the need for adjusted analysis, sample size was estimated with an effect size of 0.4 (large effect size), power of $95 \%$ and an alpha error of $5 \%$ using $\mathrm{G}$ power software $\left(\mathrm{G}^{*}\right.$ power version 3.1.2, Germany). A total of 84 participants were estimated to be divided into 3 groups. Considering a drop out of $10 \%$, the sample size finalised was 95 .

\section{Participant Selection Criteria}

Participants who were 18 years and above, willing to participate in the study, and those required tooth extraction were included. Participants who required a trans-alveolar tooth extraction, conditions that may impair wound healing (HIV/AIDS, chemotherapy, systemic steroids, chronic

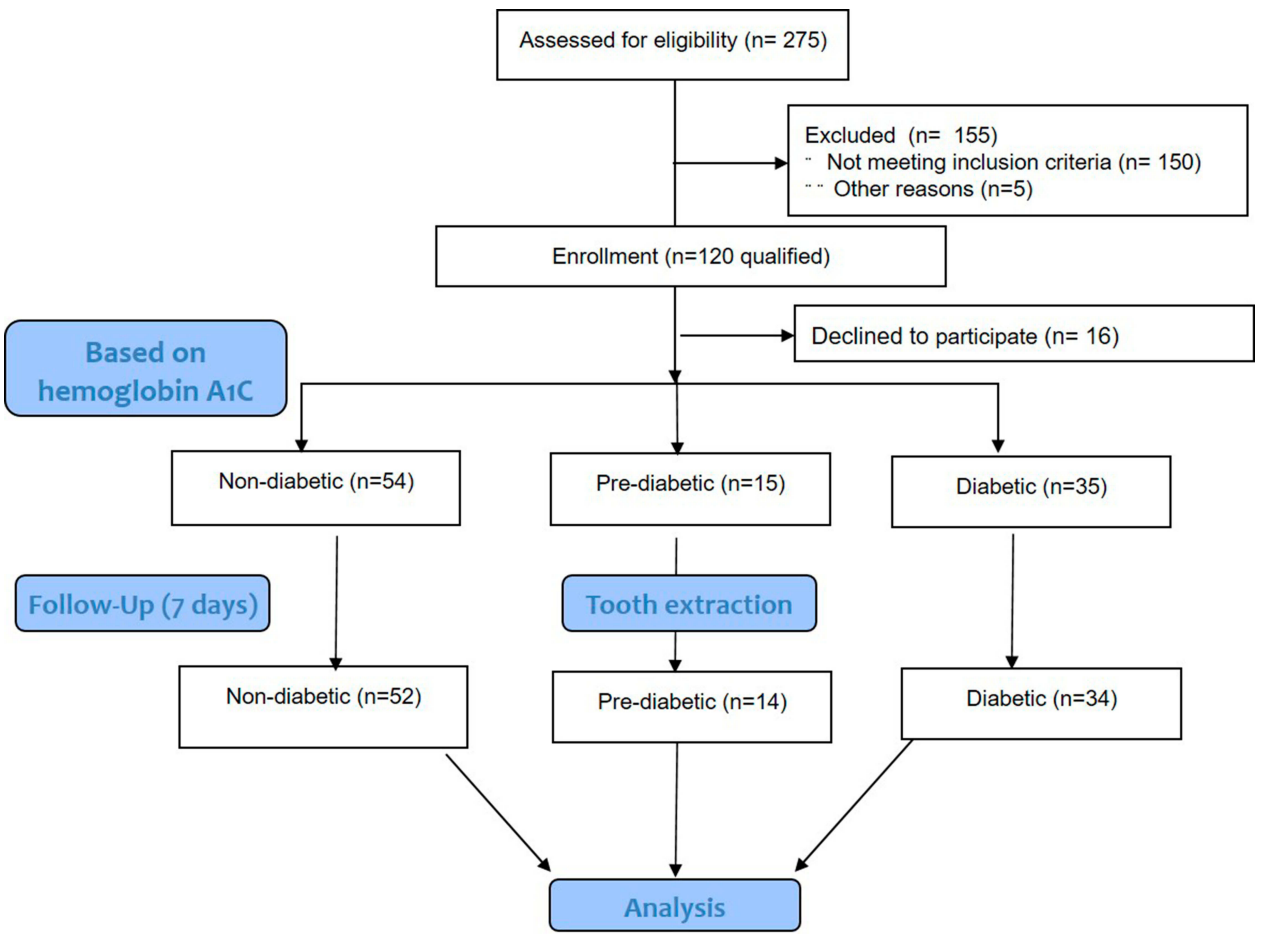

Figure I Patient flow during the study. 
alcoholics, smokers, radiotherapy, bisphosphonates, anticoagulants), benign or malignant pathology within jaws, and those unable to give consent were excluded.

\section{Data Collection}

Informed consent was obtained from all the patients. Glycated hemoglobin (HbA1c) and random blood glucose were recorded for all the participants before the procedure. Based on the HbAlc values, participants were categorised as non-diabetic, prediabetic, and diabetic. There was no scope for blinding as all the participants were familiar with their glycaemic status. However, the operator or the clinician and outcome assessor were blinded to the glycaemic status of the participants. Two trained, experienced maxillofacial surgeons performed the extractions. Patients were recalled on postoperative day 7. Sutures were not placed to approximate the mucoperiosteal flaps. Both the operators were familiar with the variables that are being studied in this research.

\section{Variables}

On the postoperative day 0 and 7 , the greatest diameter of the extraction socket was measured using a calibrated probe. Postoperative pain (PoP), discharge, swelling, infection, erythema, the occurrence of dry socket, and the number of analgesics were recorded. PoP was defined as the experience of pain during the last one week, graded as "no pain," "mild pain," "moderate," "severe," and "unbearable pain." 15 Criteria for dry socket and acute infection were recorded according to Aronovich et al. ${ }^{11}$

\section{Statistical Analysis}

All the analyses were done using SPSS version 20.0 (IBM Corp. Released 2011. IBM SPSS Statistics for Windows, Version 20.0. Armonk, NY: IBM Corp). Socket dimensions were compared using the ANOVA and ANCOVA with appropriate post hoc tests. Categorical variables were compared using the Chi-square test.

\section{Results}

A total of 100 participants completed this study, out of which $54 \%$ were male. Three patients did not return to follow-up on day 7 due to unavoidable circumstances, and one patient was excluded as the procedure was switched to transalveolar extraction. The mean age among the participants was 54.7 \pm 12.11 . No significant difference was seen with respect to age among the three groups $(\mathrm{P}=0.722)$ (Table 1). Females were significantly more in the prediabetic group than the non-
Table I Distribution of Age and Gender Among the Three Study Groups

\begin{tabular}{|l|l|l|l|}
\hline & Age & Male & Female \\
\cline { 2 - 4 } & Mean士 SD & $\mathbf{N}(\%)$ & $\mathbf{N}(\%)$ \\
\hline Group & & & \\
$\quad$ Non Diabetic & $54.04 \pm 11.52$ & $34(65.4)$ & $18(34.6)$ \\
Pre-diabetic & $57.00 \pm 10.94$ & $4(28.6)$ & $10(71.4)$ \\
Diabetic & $54.76 \pm 13.58$ & $16(47.1)$ & $18(52.9)$ \\
\hline Total & $54.7 \pm 12.11$ & $54(54)$ & $46(46)$ \\
\hline
\end{tabular}

diabetic and diabetic groups $(\mathrm{P}=0.03)$ (Table 1). All the patients in the diabetic group were on medication for oral hypoglycemics.

There was a significant decrease in the mean socket size after day 7 in all the groups when compared to the socket size on day 0 (Table 2). On day 0 , no significant difference in the mean socket size was seen among the three study groups $(\mathrm{P}=0.101)$. However, a significant difference was seen in the mean socket size on day 7. A post hoc test showed that the Diabetic group had higher socket size than the non-diabetic group $(\mathrm{P}=0.011)$.

Complications like swelling and infection were more in the diabetic group than prediabetic and non-diabetic groups (Table 3). No significant difference was seen in the distribution of pain scores among the three study groups ( $\mathrm{P}=0.312$ ) (Table 4). The mean number of analgesics consumed in non-diabetic, prediabetic, and diabetic were $7.12 \pm 1.58,7.14 \pm 1.61$, and $7.76 \pm 1.63$, respectively $(\mathrm{P}=0.169)$.

The homogeneity-of-regression (slope) assumption was evaluated for interaction between the covariate (socket dimension on day 0 ) and the factor (Group) in the prediction of the dependent variable (socket dimension on day 7). The interaction was not significant. Hence, ANCOVA was performed. The results of the ANCOVA show that the adjusted

Table 2 Intra and Intergroup Comparisons of Extraction Socket Size on Postoperative Day 0 and 7

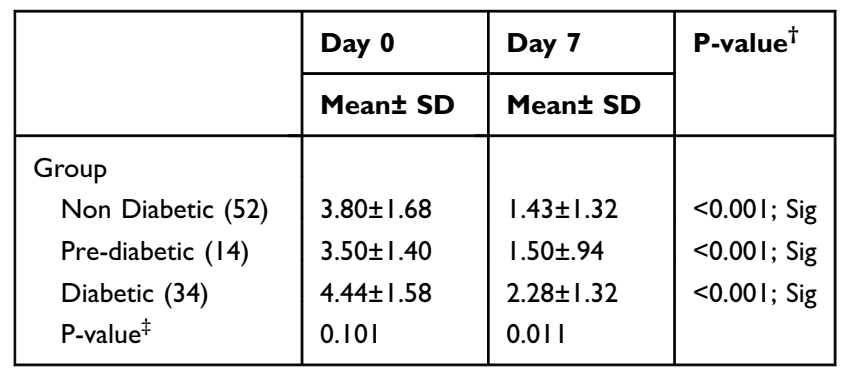

Notes: ${ }^{\dagger}$ Paired $t$-test; ${ }^{\ddagger}$ ANOVA. 
Table 3 Distribution of Complications Among the Three Study Groups

\begin{tabular}{|c|l|l|l|l|}
\hline & Swelling & Infection & Discharge & Complications \\
\cline { 2 - 5 } & $\mathbf{N}(\%)$ & $\mathbf{N}(\%)$ & $\mathbf{N}(\%)$ & $\mathbf{N}(\%)$ \\
\hline Group & & & & \\
Non-diabetic & $3(5.8)$ & $0(0)$ & $0(0)$ & $0(0)$ \\
Pre-diabetic & $\mathrm{I}(7.1)$ & $0(0)$ & $0(0)$ & $1(7.1)$ \\
Diabetic & $8(23.5)$ & $2(5.9)$ & $0(0)$ & $0(0)$ \\
\hline
\end{tabular}

Table 4 Distribution of Postoperative Pain Scores Among the Three Study Groups

\begin{tabular}{|l|l|l|l|l|}
\hline & Mild & Moderate & Severe & \multirow{2}{*}{ P-value } \\
\cline { 2 - 4 } & $\mathbf{N}(\%)$ & $\mathbf{N}(\%)$ & $\mathbf{N}(\%)$ & \\
\hline $\begin{array}{l}\text { Group } \\
\text { Non-diabetic }\end{array}$ & $34(65.4)$ & $13(25.0)$ & $5(9.6)$ & \multirow{2}{*}{0.312} \\
$\begin{array}{l}\text { Pre-diabetic } \\
\text { Diabetic }\end{array}$ & $8(57.1)$ & $6(42.9)$ & $0(0)$ & \\
\hline
\end{tabular}

means concerning the group was significant $(\mathrm{P}=0.043)$ (Figure 2). A post hoc test showed that the adjusted means were significantly higher for diabetic than the non-diabetic group. The effect size for this difference was moderate $(0.57)$.

\section{Discussion}

Management of diabetic patients is often an uphill task for the oral health care providers. Oral and maxillofacial surgeons often experience challenging situations in the management of diabetic patients. The present study compared the clinical outcomes after extraction among diabetic, prediabetic, and non-diabetic groups.

In the present study, the diabetic group was found to have a larger socket size than the non-diabetic group on postoperative day 7 , which suggested a slow healing process than non-diabetic and prediabetic patients. It was evident with additional analysis after adjusting the socket size of postoperative day 0 . Thus, the finding of the study supported the fact that higher the glycemic levels could delay healing. However, contrary results have been reported in the literature. Aronovich et al reported that preoperative glucose and HbAlc levels do not influence the healing of the extraction socket. ${ }^{11}$ Fernandes et al reported lack of correlation between delay in epithelization and glycemic control. ${ }^{12}$ Few researchers reported that there was no significant difference in the healing between diabetics and controls. ${ }^{10,13}$

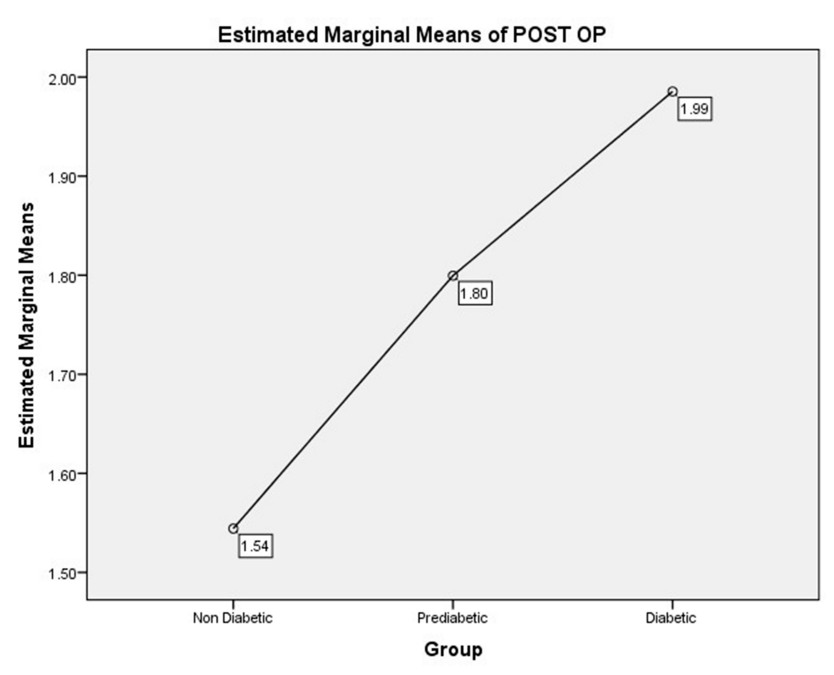

Covariates appearing in the model are evaluated at the following values: PRE OP SOCKET SIZE $=3.9750$

Figure 2 Comparison of estimated marginal means of extraction socket size on postoperative day 7 in the three study groups adjusted for extraction socket size on postoperative day 0 .

The present study also assessed that factors like swelling, infection were more likely to be seen in people with diabetes than non-diabetics and prediabetics. The overall postoperative pain experience was also similar among all the groups. Overall, the extraction sockets healed without persistent complications. Similar findings were evident in previous studies that reported minimal complications without postoperative infections, osteomyelitis, or osteonecrosis. ${ }^{10,12}$

There is substantial variation in methodology among the studies conducted in the past. Lack of standardized control group, ${ }^{11}$ combined type 1 and 2 diabetes patients, ${ }^{11}$ different operators performing the extraction, ${ }^{11}$ variable follow-up rates ( 1 week to 60 days $)^{10-13}$ were some of the factors.

There were some limitations in our study as well. The unequal distribution of the patients in each group could have led to confounding. Hence, socket dimensions of day 7 were adjusted to address the confounding and the result was reported as estimated marginal means. The recall period in our study was short (1 week) but was similar to studies done earlier. ${ }^{10,13}$ This was decided to minimize the attrition of patients and optimize the limited resources. The number of days required for complete closure of the socket would have provided some valuable insight into the actual time of healing. But such a variable would need more frequent multiple follow-ups leading to increased attrition rates and may not be feasible in low resource settings. Future studies should include Ecological momentary assessment of extraction 
socket along with the patient-reported outcomes. A systematic review highlighted essential aspects of the management of dental extraction in diabetic patients which include scheduling of appointments in the morning, extractions with meal times, monitoring of blood glucose for permissible limits, and use of antibiotics after extraction. ${ }^{14}$

All the diabetic participants in the study were on medication with a lot of variation in the drug type, frequency, and dosages of the medications. Due to the nature of the study, it was not possible to have a control of the medications on the healing. These medications have a role in the effective management of the glycemic status of the participant which may have a potential role in the healing or outcomes after extraction. There would be an overall favourable result towards the healing of the extraction sockets due to the medication that maintains glycemic level.

This study used a pragmatic approach to evaluate the healing of extraction sockets among the diabetics who were on medication. Uncontrolled diabetics and known diabetics who were not on medication are out of the scope of our study as we do not offer the same standard of care in our institution. Unlike explanatory trials, pragmatic trials are the ones that are done under routine clinical conditions. They have high external validity with simple design and diverse settings. This study followed such a pragmatic approach to evaluate any adverse events due to the extraction of teeth in diabetics. This study would help general dental practitioners in the decision process (extraction or defer) keeping in view of the complications and healing in people with diabetes.

\section{Conclusion}

Within the limits of the study, socket dimensions were larger on postoperative day 7 in people with diabetes when compared to non-diabetics which suggested delayed healing. The incidence of complications was minimal in optimally controlled diabetic patients. Empirical antibiotics, appropriate care, and monitoring are required in patients who have an additional risk of postoperative infections and delayed healing.

\section{Statement of Ethics}

This research complies with the guidelines for human studies and the research was conducted ethically in accordance with the World Medical Association Declaration of Helsinki.

\section{Acknowledgments}

We thank all the participants and the non-teaching staff of our department.

\section{Author Contributions}

SG, KS-Conceptualization, data acquisition, initial draft and final draft. KCP - Conceptualization, data acquisition and curation, formal analysis, interpretation, initial draft and final draft. SR, JR, AW - Conceptualization, methodology, data acquisition, initial draft, final draft, review of the literature, interpretation. All authors agreed on all the versions of the manuscript, journal to which the article will be submitted, take responsibility and be accountable for the contents of the article and contributed to the important intellectual content.

\section{Funding}

There is no funding to report.

\section{Disclosure}

The authors report no conflicts on interest in this work.

\section{References}

1. World Health Organization. Definition, Diagnosis and Classification of Diabetes Mellitus and Its Complications: Report of a WHO Consultation. Part 1, Diagnosis and Classification of Diabetes Mellitus. World Health Organization; 1999.

2. WHO Study Group on Prevention of Diabetes Mellitus \& World Health Organization. Prevention of Diabetes Mellitus: Report of a WHO Study Group. World Health Organization; 1994.

3. Kaveeshwar S, Cornwall J. The current state of diabetes management. Australas Med J. 2014;7(1):45-48. doi:10.4066/AMJ.2013.1979

4. Joshi SR, Parikh RM. India - Diabetes capital of the world: now heading towards hypertension. $J$ Assoc Physicians India. 2007;55 (MAY):323-324.

5. Ekmektzoglou KA, Zografos GC. A concomitant review of the effects of diabetes mellitus and hypothyroidism in wound healing. World $J$ Gastroenterol. 2006;12(17):2721-2729. doi:10.3748/wjg.v12.i17.2721

6. Lioupis C. Effects of diabetes mellitus on wound healing: an update. $J$ Wound Care. 2005;14(2):84-86. doi:10.12968/jowc.2005.14.2.26738

7. Khan SQ, Khabeer A, Al-Thobity AM, Benrashed MA, Alyousef NI, AlMaimouni Y. Correlation between diabetes mellitus and number of restored, carious lesions and missing teeth: a retrospective radiographic evaluation. Saudi Dent J. 2020. doi:10.1016/j.sdentj.2019.12.006

8. Nishimura F, Takahashi K, Kurihara M, Takashiba S, Murayama Y. Periodontal disease as a complication of diabetes mellitus. Ann Periodontol. 1998;3(1):20-29. doi:10.1902/annals.1998.3.1.20

9. Grossi SG, Genco RJ. Periodontal disease and diabetes mellitus: a two-way relationship. Ann Periodontol. 1998;3(1):51-61. doi:10. 1902/annals.1998.3.1.51

10. Huang S, Dang H, Huynh W, Sambrook PJ, Goss AN. The healing of dental extraction sockets in patients with type 2 diabetes on oral hypoglycaemics: a prospective cohort. Aust Dent J. 2013;58(1):8993. doi:10.1111/adj.12029

11. Aronovich S, Skope LW, Kelly JPW, Kyriakides TC. The relationship of glycemic control to the outcomes of dental extractions. J Oral Maxillofac Surg. 2010;68(12):2955-2961. doi:10.1016/j.joms.2010. 05.006 
12. Fernandes KS, Glick M, De Souza MS, Kokron CM, Gallottini M. Association between immunologic parameters, glycemic control, and postextraction complications in patients with type 2 diabetes. $J \mathrm{Am}$ Dent Assoc. 2015;146(8):592-599. doi:10.1016/j.adaj.2015.02.014

13. Power DJ, Sambrook PJ, Goss AN. The healing of dental extraction sockets in insulin-dependent diabetic patients: a prospective controlled observational study. Aust Dent J. 2019;64(1):111-116. doi:10.1111/adj.12669
14. Gazal G. Management of an emergency tooth extraction in diabetic patients on the dental chair. Saudi Dent J. 2020;32(1):1-6. doi:10.1016/j.sdentj.2019.07.004

15. Ferraz MB, Quaresma MR, Aquino LRL, Atra E, Tugwell P, Goldsmith CH. Reliability of pain scales in the assessment of literate and illiterate patients with rheumatoid arthritis. J Rheumatol. 1990;17 (8):1022-1024.

\section{Publish your work in this journal}

Clinical, Cosmetic and Investigational Dentistry is an international, peer-reviewed, open access, online journal focusing on the latest clinical and experimental research in dentistry with specific emphasis on cosmetic interventions. Innovative developments in dental materials, techniques and devices that improve outcomes and patient satisfaction and preference will be highlighted. The manuscript management system is completely online and includes a very quick and fair peer-review system, which is all easy to use. Visit http://www.dovepress.com/testimonials.php to read real quotes from published authors. 